\title{
BMJ Open A protocol for a prospective observational study using chest and thumb ECG: transient ECG assessment in stroke evaluation (TEASE) in Sweden
}

Peter Magnusson, ${ }^{1,2}$ Hirsh Koyi, ${ }^{2}$ Gustav Mattsson ${ }^{2}$

To cite: Magnusson P, Koyi H, Mattsson G. A protocol for a prospective observational study using chest and thumb ECG: transient ECG assessment in stroke evaluation (TEASE) in Sweden. BMJ Open 2018;8:e019933. doi:10.1136/ bmjopen-2017-019933

- Prepublication history and additional material for this paper are available online. To view these files, please visit the journal online (http://dx.doi. org/10.1136/bmjopen-2017019933).

Received 5 0ctober 2017 Revised 19 December 2017 Accepted 8 February 2018

\section{Check for updates}

${ }^{1}$ Cardiology Research Unit, Department of Medicine, Karolinska Institutet, Stockholm, Sweden

${ }^{2}$ Centre for Research and Development, Uppsala University/Region Gävleborg, Gävle, Sweden

Correspondence to Dr Peter Magnusson; peter.magnusson@ regiongavleborg.se

\section{ABSTRACT}

Introduction Atrial fibrillation (AF) causes ischaemic stroke and based on risk factor evaluation warrants anticoagulation therapy. In stroke survivors, AF is typically detected with short-term ECG monitoring in the stroke unit. Prolonged continuous ECG monitoring requires substantial resources while insertable cardiac monitors are invasive and costly. Chest and thumb ECG could provide an alternative for AF detection poststroke. The primary objective of our study is to assess the incidence of newly diagnosed AF during 28 days of chest and thumb ECG monitoring in cryptogenic stroke. Secondary objectives are to assess health-related quality of life (HRQoL) using shortform health survey (SF-36) and the feasibility of the Coala Heart Monitor in patients who had a stroke.

Methods Stroke survivors in Region Gävleborg, Sweden, will be eligible for the study from October 2017. Patients with a history of ischaemic stroke without documented AF before or during ECG evaluation in the stroke unit will be evaluated by the chest and thumb ECG system Coala Heart Monitor. The monitoring system is connected to a smartphone application which allows for remote monitoring and prompt advice on clinical management. Over a period of 28 days, patients will be monitored two times a day and may activate the ECG recording at symptoms. On completion, the system is returned by mail. This system offers a possibility to evaluate the presence of AF poststroke, but the feasibility of this system in patients who recently suffered from a stroke is unknown. In addition, HRQoL using SF-36 in comparison to Swedish population norms will be assessed. The feasibility of the Coala Heart Monitor will be assessed by a self-developed questionnaire.

Ethics and dissemination The study was approved by The Regional Ethical Committee in Uppsala (2017/321). The database will be closed after the last follow-up, followed by statistical analyses, interpretation of results and dissemination to a scientific journal.

Trial registration number NCT03301662; Pre-results.

\section{INTRODUCTION}

Atrial fibrillation (AF) causes stroke and systemic embolisation, but these devastating events can be prevented by anticoagulant therapy. ${ }^{1}$ A non-vitamin $\mathrm{K}$ antagonist oral anticoagulant (NOAC) is the preferred

\section{Strengths and limitations of this study}

- Chest and thumb ECG evaluation applied after 24hour ECG Holter at the stroke unit after the confirmation of ischaemic stroke.

- Usage of a smartphone application for the storage of chest and thumb ECG which may improve compliance and facilitate fast evaluation by the healthcare provider in order to prescribe an anticoagulant when indicated.

- Prospective design including consecutive patients which eliminate both selection and tertiary centre bias.

- Management of short (30 s) atrial fibrillation episodes, detected by non-12-lead ECG, lack evidence from randomised controlled trials with regard to benefit of anticoagulation.

- The small sample size $(n=100)$ may imply type-II errors in subgroup analyses.

choice and effectively reduce the risk of stroke and mortality. ${ }^{2}$ A meta-analysis of the pivotal NOAC trials showed a $19 \%$ reduction of stroke/systemic embolism and $10 \%$ lower mortality compared with warfarin. ${ }^{2}$ If $\mathrm{AF}$ is not diagnosed, antiplatelet medication is current practice following a stroke. ${ }^{3}$ According to the European Society of Cardiology (ESC), antiplatelet monotherapy should not be considered in the presence of $\mathrm{AF}$, regardless of the stroke risk..$^{4-6}$ Stroke is a leading cause of disability and death, and the incidence is increasing due to ageing populations and the growing prevalence of risk factors such as diabetes and hypertension. ${ }^{7-9}$ At least $20 \%-30 \%$ of patients with ischaemic stroke have a documented episode of $\mathrm{AF}$ before, during or after the event, but in a quarter of these patients, the stroke is cryptogenic, meaning that no aetiological factor can be determined. ${ }^{10-12}$ However, the proportion of cryptogenic stroke from studies varies due to heterogeneity of cohorts and evaluation tools. 
Possibilities for AF detection include monitoring on the hospital ward, repeated ECG, Holter monitoring, external event or loop recorders and long-term outpatient monitoring. Insertable cardiac monitors in cryptogenic stroke yield an AF diagnosis in $8.9 \%$ at 6 months and $12.4 \%$ at 12 months, but this strategy has not been endorsed in current practice as it requires considerable resources and imply high costs, even though cost-effectiveness has been suggested. ${ }^{61314}$ Episodes of AF may be silent, thus not recognised or reported by the patient, but are nevertheless associated with the same risk of embolisation. ${ }^{15-17}$ In patients with either dual-chamber pacemakers or implantable defibrillators and with no documented history of AF, $10.1 \%$ had episodes of highrate atrial tachycardia, and this was also associated with an increased risk of stroke. ${ }^{18}$

Sequential stratified ECG monitoring detected $\mathrm{AF}$ in $24 \%$ of patients who had a stroke in one study. ${ }^{10}$ The diagnostic yield was $11.5 \%$ in a pooled analysis, but this yield varies with such study factors as timing, length of registration and the monitoring tool. ${ }^{19}$ In unselected stroke patients , 24-hour monitoring found AF in only 2.4\%. ${ }^{20}$ This may vary substantially with the recording technique; in a recent study, AF (defined as $\geq 30 \mathrm{~s}$ in duration) was detected in $16.1 \%$ of patients monitored by a 30 day event-triggered recorder compared with $3.2 \%$ of patients monitored by 24-hour ECG. ${ }^{21}$ A large multicentre study of patients who had a stroke on Holter monitoring reported new diagnoses of AF in $2.6 \%$ of patients at 24 hours and $4.3 \%$ at 72 hours. ${ }^{12}$ In another study, AF was detected in $8.3 \%$ of patients who had a stroke monitored by continuous ECG for a median of 89 hours in the stroke unit; ECG monitoring was superior to 24-hour Holter monitoring in detecting $\mathrm{AF}^{22}$

Thus, while ECG monitoring for an extended period is important for stroke survivors postdischarge, Holter monitoring is impractical, ECG data storage is limited and data interpretation requires considerable resources. Therefore, the thumb ECG offers advantages in that it monitors conveniently (typically two times a day) and can be activated to capture symptomatic episodes. For example, AF was detected in $11.4 \%$ of post-stroke patients monitored by thumb ECG over 21 days versus $2.8 \%$ in those continuously monitored for 48 hours. ${ }^{23}$

The thumb ECG monitor system Zenicor (Zenicor Medical Systems AB, Stockholm, Sweden) has been shown to diagnose previously unknown $\mathrm{AF}$ in $3.0 \%$ of the general population in Sweden aged 75 years. ${ }^{24}$ The Zenicor system has been developed and integrated into the chest and thumb ECG Coala Heart Monitor (Coala Life AB, Stockholm, Sweden). The monitoring system uses a smartphone application which allows for remote monitoring by a clinician. This system may help to evaluate the presence of AF poststroke. However, the feasibility of this system in patients who recently suffered a stroke has not been studied. In addition, health-related quality of life (HRQoL) using short-form health survey (SF-36) in comparison to Swedish population norms will be assessed. ${ }^{25}{ }^{26}$ The feasibility of the Coala Heart Monitor will be assessed by a self-developed questionnaire (see online supplementary file 1 ).

\section{OBJECTIVES}

The primary objective is to assess the incidence of newly diagnosed AF during 28 days of chest and thumb ECG in patients who had a cryptogenic stroke.

The secondary objectives are to assess HRQoL using SF-36 and the feasibility of the Coala Heart Monitor in patients who had a stroke. In addition, patients who had a stroke, not eligible for the chest and thumb ECG monitoring, will be analysed with regard to prevalence of previous atrial arrhythmia (including whether they were anticoagulated), cumulative incidence of stroke after 3 years and all-cause mortality after 3 years in patients with $\mathrm{AF}$ versus no $\mathrm{AF}$.

\section{METHODS}

\section{Setting and selection}

Patients with a clinically confirmed diagnosis of ischaemic stroke will be recruited from the catchment area of Region Gävleborg, Sweden. Eligible patients will be identified from daily checks of the medical records in the stroke unit. The recruitment is planned to start in October 2017.

\section{Inclusion and exclusion}

Patients, aged $\geq 18$ years, with a validated diagnosis of ischaemic cryptogenic stroke are eligible for the study. Cryptogenic stroke is defined as cerebral ischaemia of unknown aetiology, that is, not attributable to a source of cardiac embolism, large artery atherosclerosis or small artery disease despite a standard vascular, cardiac and serological evaluation. ${ }^{27}$ For screening with chest and thumb ECG, exclusion criteria are as follows: previously known atrial arrhythmia with an indication for anticoagulation, implantable defibrillator, pacemaker or insertable cardiac monitor, pregnancy, permanent indication for anticoagulation (including low-molecular-weight heparin) due to atrial arrhythmia, mechanical heart valve, deep vein thrombosis or pulmonary embolism. Patients with a life expectancy $\leq 6$ months (eg, severe heart failure New York Heart Association functional class IV or malignancy) are likewise excluded.

\section{Variables}

Atrial arrhythmia is defined as AF, atrial flutter or ectopic atrial tachycardia with a duration of at least $30 \mathrm{~s}$. Patients characteristics are age, sex, date of current ischaemic stroke, previous stroke, known AF, medication (warfarin, NOAC, antiplatelet therapy), heart failure, hypertension, diabetes mellitus, vascular disease (peripheral vascular disease, aortic plaque, coronary artery disease), National Institutes of Health Stroke Scale, 12-lead ECG, and when applicable imaging 
from carotid-Doppler, CT, echocardiography, or transoesophageal echocardiography, as well as coagulation laboratory examination.

\section{Outcome measurements}

Outcome measurements are arrhythmias recorded by chest and thumb ECG obtained during scheduled two times a day recordings or by patient-activated recordings.

Each episode will be classified as AF, atrial flutter, ectopic atrial tachycardia, ventricular tachycardia, premature ventricular complex or supraventricular ventricular complex. The date and time of each episode will be recorded.

\section{Research questions and endpoints}

The primary endpoint is 28-day cumulative incidence of atrial arrhythmia at 28 days.

\section{Secondary endpoints}

a. Prevalence of previously known atrial arrhythmia before the inclusion in the study and the number of these patients who had anticoagulant therapy.

b. Compliance with chest and thumb ECG at week 4 (number of recorded scheduled ECG tracings).

c. Patient-reported experience with chest and thumb ECG measured at week 6 (questionnaire as online supplementary file 1 ).

d. HRQoL (SF-36) at week 6 and at 12 months and the association with $\mathrm{AF}$ and compliance with chest and thumb ECG.

e. Cumulative incidence of stroke after 3 years in patients with $\mathrm{AF}$ versus without $\mathrm{AF}$.

f. All-cause mortality after 3 years in patients with $\mathrm{AF}$ versus no $\mathrm{AF}$.

\section{Chest and thumb ECG}

Patients will be asked to use the chest and thumb ECG monitor device two times a day, once between 06:00 and 10:00 hours and again between 18:00 and 22:00 hours. The monitoring will start within a few days when the diagnosis of stroke has been confirmed and standard evaluation is complete, typically $1-5$ days.

If the patients feel palpitations or other symptoms suggestive of arrhythmia (eg, sudden onset of tiredness, presyncope, syncope), they are asked to record the episode with the smartphone application. Each patient is monitored for four consecutive weeks, after which the device is returned by mail to the investigators.

Each recording is stored in a web-based application that is accessible to the investigators. The investigators daily check all recordings. In the case of an AF episode, we contact the patient (or relative/healthcare provider) as soon as possible, typically the same day. The reason for this is that they require anticoagulation and they typically need prompt protection (time is recorded). In the case of an AF episode, two investigators, of whom one is an experienced cardiologist within the field of arrhythmia, interpret the recording.

\section{Power analysis}

A power analysis ${ }^{28}$ based on previous research findings and estimation of outcome to $2.4 \%, 95 \%$ CI, width of CI $5 \%$, SD $12 \%$ results in a sample size of 89 . There is likely to be dropout of patients who are unable or unwilling to meet the monitoring requirements; thus, an estimated 120 patients should be included in order to have 100 patients complete the chest and thumb ECG evaluation.

\section{Statistics}

Descriptive data will be reported as frequencies, percentages, means and percentiles. Continuous variables are summarised as means, SDs and percentiles, and t-tests for group comparisons, while $\chi^{2}$ test is used for categorical variables. Kaplan-Meier estimates are used to describe time to event analysis, and cumulative incidence at 1,2 , 3 and 4 weeks will be reported. Statistical significance is defined as a two-sided $p$ value of $<0.05$. The data will be stored in Excel 2010 (Microsoft) and imported into SPSS V.22 (IBM) for analyses.

\section{Ethics and dissemination}

The study protocol, including variables and prespecified research questions, were registered at Clinical Trial Registration NCT03301662 and approved on 3 October 2017. The documentation of research data and management of the study follow the Guideline for Good Clinical Practice. ${ }^{29}$ Each patient is informed about the study by a physician and nurse and included after written consent. After the study is completed, the database will be closed and followed by statistical analyses, interpretation of results and dissemination to scientific journals.

\section{DISCUSSION}

Since anticoagulation therapy has been proven effective in preventing ischaemic stroke in patients with $\mathrm{AF}$, reliable AF detection following cryptogenic stroke is crucial. ${ }^{6}$ Hence, prolonged ECG monitoring is reasonable, especially in patients at high risk of embolisation. Ischaemic stroke risk stratification and the decision to prescribe anticoagulants is based on $\mathrm{CHA}_{2} \mathrm{DS}_{2}$-VASc scores; a prior history of stroke counts for two points, which suffices as a rationale for anticoagulation. ${ }^{6}$ The vast majority of patients who had a stroke typically have multiple risk factors, and stroke risk increases with more risk factors. ${ }^{30}$ ESC guidelines already allow for prolonged monitoring of these patients: 'In stroke patients, additional ECG monitoring by long-term noninvasive or implanted loop recorders should be considered to document silent atrial fibrillation' (class IIa recommendation, level of evidence B). ${ }^{6}$ However, since the Cryptogenic Stroke and Underlying $\mathrm{AF}$ trial, ${ }^{13}$ current practice in Sweden remains unchanged with invasive monitoring rarely used for $\mathrm{AF}$ detection in patients who had a stroke and still not endorsed by national authorities. ${ }^{31}$ The non-invasive thumb ECG has been advocated in that it provides an alternative and advantageous cost-benefit profile in 
mass screening. ${ }^{32}$ Patients who had a stroke have a higher risk for recurrent stroke and higher incidences of $\mathrm{AF}$, so non-invasive thumb ECG monitoring may be of even greater benefit in this population. This has yet to be analysed, and it is our hope that our study will advance the knowledge of thumb ECG in this population particularly with regard to healthcare economics.

The use of the thumb ECG has been studied in a Swedish setting for patients who had a stroke, but the study was retrospective, with data gathered at different times after the stroke, and the monitoring method was selected based on the physician's preference, which implies bias. Our prospective study includes consecutive stroke patients without referral centre bias. ${ }^{33}$ This will provide a basis to estimate AF incidence over an extended period of 28 days. Continuous Holter monitoring may be associated with poor compliance, technical difficulties and time-consuming analyses of extensive amounts of data by healthcare providers. ${ }^{33}$ The newly developed Coala Heart Monitor with the proven detection algorithms from Zenicor using a smartphone application seems to be a promising alternative, but feasibility remains to be studied. Therefore, we added a questionnaire to address feasibility issues.

Although a thumb ECG may provide an attractive method of non-invasive $\mathrm{AF}$ detection, there remain some controversies with regard to anticoagulation for short-term AF. The potential benefits of anticoagulation therapy for short-term AF would be challenging to study because it would require long-term follow-up, demands a large sample size and raises ethical concerns about withholding anticoagulation from a stroke survivor. This proposed prospective observational trial of consecutive stroke patients using thumb ECG has the prerequisites to evaluate outcome at 28 days and analyse the clinical feasibility of the Coala Heart Monitor.

Acknowledgements The authors acknowledge editing by Jo Ann LeQuang of LeQ Medical who reviewed the manuscript for American English. Ulf Tossman, David Fällman and Philip Siberg from Coala Life for support regarding the Coala Life Monitor.

Contributors PM: idea, design, project management and writing of the manuscript. HK: critical revision. GM: idea, design, project management and critical revision.

Funding Region Gävleborg funded this research project and Coala Life provided free product Coala Heart Monitor during the study period.

Competing interests The authors received free product from Coala Life.

Patient consent Not required.

Ethics approval The study was approved by the Regional Ethical committee in Uppsala (Dnr 2017/321).

Provenance and peer review Not commissioned; externally peer reviewed.

Open Access This is an Open Access article distributed in accordance with the Creative Commons Attribution Non Commercial (CC BY-NC 4.0) license, which permits others to distribute, remix, adapt, build upon this work non-commercially, and license their derivative works on different terms, provided the original work is properly cited and the use is non-commercial. See: http://creativecommons.org/ licenses/by-nc/4.0/

(C) Article author(s) (or their employer(s) unless otherwise stated in the text of the article) 2018. All rights reserved. No commercial use is permitted unless otherwise expressly granted.

\section{REFERENCES}

1. Hart RG, Pearce LA, Aguilar MI. Meta-analysis: antithrombotic therapy to prevent stroke in patients who have nonvalvular atrial fibrillation. Ann Intern Med 2007;146:857-67.

2. Ruff CT, Giugliano RP, Braunwald E, et al. Comparison of the efficacy and safety of new oral anticoagulants with warfarin in patients with atrial fibrillation: a meta-analysis of randomised trials. Lancet 2014;383:955-62.

3. Kernan WN, Ovbiagele B, Black HR, et al. Guidelines for the prevention of stroke in patients with stroke and transient ischemic attack: a guideline for healthcare professionals from the American Heart Association/American Stroke Association. Stroke 2014;45:2160-236.

4. Olesen JB, Lip GY, Lindhardsen J, et al. Risks of thromboembolism and bleeding with thromboprophylaxis in patients with atrial fibrillation: A net clinical benefit analysis using a 'real world' nationwide cohort study. Thromb Haemost 2011;106:739-49.

5. Själander S, Själander A, Svensson PJ, et al. Atrial fibrillation patients do not benefit from acetylsalicylic acid. Europace 2014;16:631-8.

6. Kirchhof P, Benussi S, Kotecha D, et al. 2016 ESC Guidelines for the management of atrial fibrillation developed in collaboration with EACTS. Eur Heart J 2016;37:2893-962.

7. Schnabel RB, Yin X, Gona P, et al. 50 year trends in atrial fibrillation prevalence, incidence, risk factors, and mortality in the Framingham Heart Study: a cohort study. Lancet 2015;386:154-62.

8. Andersson T, Magnuson A, Bryngelsson IL, et al. All-cause mortality in 272,186 patients hospitalized with incident atrial fibrillation 19952008: a Swedish nationwide long-term case-control study. Eur Heart J 2013;34:1061-7.

9. Go AS, Mozaffarian D, Roger VL, et al. Heart disease and stroke statistics--2014 update: a report from the American Heart Association. Circulation 2014;129:e28-e292.

10. Kishore A, Vail A, Majid A, et al. Detection of atrial fibrillation after ischemic stroke or transient ischemic attack: a systematic review and meta-analysis. Stroke 2014;45:520-6.

11. Henriksson KM, Farahmand B, Åsberg S, et al. Comparison of cardiovascular risk factors and survival in patients with ischemic or hemorrhagic stroke. Int J Stroke 2012;7:276-81.

12. Grond M, Jauss M, Hamann G, et al. Improved detection of silent atrial fibrillation using 72-hour Holter ECG in patients with ischemic stroke: a prospective multicenter cohort study. Stroke 2013;44:3357-64.

13. Sanna T, Diener HC, Passman RS, et al. Cryptogenic stroke and underlying atrial fibrillation. N Engl J Med 2014;370:2478-86.

14. Diamantopoulos A, Sawyer LM, Lip GY, et al. Cost-effectiveness of an insertable cardiac monitor to detect atrial fibrillation in patients with cryptogenic stroke. Int J Stroke 2016;11:302-12.

15. Savelieva I, Camm AJ. Clinical relevance of silent atrial fibrillation: prevalence, prognosis, quality of life, and management. J Interv Card Electrophysiol 2000;4:369-82.

16. Friberg L, Hammar N, Rosenqvist M. Stroke in paroxysmal atrial fibrillation: report from the Stockholm Cohort of Atrial Fibrillation. Eur Heart J 2010;31:967-75.

17. Vanassche T, Lauw MN, Eikelboom JW, et al. Risk of ischaemic stroke according to pattern of atrial fibrillation: analysis of 6563 aspirin-treated patients in ACTIVE-A and AVERROES. Eur Heart $J$ 2015;36:281-8

18. Healey JS, Connolly SJ, Gold MR, et al. Subclinical atrial fibrillation and the risk of stroke. N Engl J Med 2012;366:120-9.

19. Sposato LA, Cipriano LE, Saposnik G, et al. Diagnosis of atrial fibrillation after stroke and transient ischaemic attack: a systematic review and meta-analysis. Lancet Neurol 2015;14:377-87.

20. Shafqat S, Kelly PJ, Furie KL, et al. Holter monitoring in the diagnosis of stroke mechanism. Intern Med J 2004;34:305-9.

21. Gladstone DJ, Spring M, Dorian P, et al. Atrial fibrillation in patients with cryptogenic stroke. N Engl J Med 2014;370:2467-77.

22. Rizos T, Güntner J, Jenetzky E, et al. Continuous stroke unit electrocardiographic monitoring versus 24-hour Holter electrocardiography for detection of paroxysmal atrial fibrillation after stroke. Stroke 2012;43:2689-94.

23. Orrsjö G, Cederin B, Bertholds E, et al. Screening of paroxysmal atrial fibrillation after ischemic stroke: 48-hour holter monitoring versus prolonged intermittent ECG recording. ISRN Stroke 2014;2014:1-6. 6 pages.

24. Friberg L, Engdahl J, Frykman V, et al. Population screening of 75- and 76-year-old men and women for silent atrial fibrillation (STROKESTOP). Europace 2013;15:135-40.

25. McHorney CA, Ware JE, Lu JF, Jf L, et al. The MOS 36-item short-form health survey (SF-36): III. Tests of data quality, scaling assumptions, and reliability across diverse patient groups. Med Care 1994;32:40-66. 
26. Sullivan M, Karlsson J, Ware JE. The Swedish sf-36 health survey- I. evaluation of data quality, scaling assumptions, reliability and construct validity across general populations in Sweden. Soc Sci Med 1995;41:1349-58.

27. Adams HP, Bendixen BH, Kappelle LJ, et al. Classification of subtype of acute ischemic stroke. Definitions for use in a multicenter clinical trial. TOAST. Trial of Org 10172 in Acute Stroke Treatment. Stroke 1993;24:35-41.

28. Hulley SB, Cummings SR, Browner WS, et al. Designing clinical research: an epidemiologic approach. 4th edn: Philadelphia, PA: Lippincott Williams \& Wilkins, 2013:80. Appendix 6D.

29. European Medicines Agency. ICH harmonised tripartite guideline E6. In: EMA, ed. Note for guidance on good clinical practice, 2002. PMP/ $\mathrm{ICH} / 135 / 95$.
30. Gage BF, Waterman AD, Shannon W, et al. Validation of clinical classification schemes for predicting stroke: results from the National Registry of Atrial Fibrillation. JAMA 2001;285:2864-70.

31. Socialstyrelsen. National guidelines for cardiac care. www.socialstyr elsen.se/nationellariktlinjerhjartsjukvard (accessed 8 Sep 2017).

32. Levin LÅ, Husberg M, Sobocinski PD, et al. A cost-effectiveness analysis of screening for silent atrial fibrillation after ischaemic stroke. Europace 2015;17:207-14.

33. Stahrenberg R, Weber-Krüger M, Seegers J, et al. Enhanced detection of paroxysmal atrial fibrillation by early and prolonged continuous holter monitoring in patients with cerebral ischemia presenting in sinus rhythm. Stroke 2010:41:2884-8.

34. Seet RC, Friedman PA, Rabinstein AA. Prolonged rhythm monitoring for the detection of occult paroxysmal atrial fibrillation in ischemic stroke of unknown cause. Circulation 2011;124:477-86. 